\title{
Síndrome de Pusher em Pacientes com AVC e Sua Associaçáa com Gravidade Clínica e Dependência Funcional
}

\author{
Patients with Pusher Syndrome and the Association with Clinical Severity and Functional Dependence \\ Suzana Palmini', Jaderson Costa ${ }^{2}$, Magali Grave
}

\begin{abstract}
RESUMO
Objetivo. Estabelecer a prevalência da sindrome de Pusher (SP) em pacientes após acidente vascular cerebral (AVC) a partir da Scale for Contraversive Pushing (SCP - avaliaçấo do sintoma de empurrar) e correlacioná-la com anormalidades do exame neurológico, gravidade do AVC e funcionalidade. Método. Estudo transversal com amostra de conveniência, diagnóstico de AVC agudo, clinicamente estáveis e com possibilidade de avaliação da severidade do evento através da escala National Institute of Health Stroke Scale (NIHSS) e do Índice de Barthel (IB). Para o diagnóstico da SP, utilizou-se dois critérios de pontuação, com diferentes pontos de corte, na SPC: resultado maior ou igual a um (critério I) ou maior que zero (critério II). Resultados. 30 foram incluídos no estudo. As médias do NIHSS e do IB foram de 8,5 e 48,8 pontos, respectivamente. A presença de SP associou-se significativamente a valores mais baixos no IB quando se utilizou o critério II $(22,5 \pm 8,5$ versus $58,4 \pm 27,3 ; \mathrm{P}<0,001)$. Conclusáo. A prevalência da SP em paciente pós-AVC agudo é significativa e pode variar de acordo com os critérios utilizados. Sua presença associa-se a parâmetros clínicos de maior gravidade e dependência funcional, maior incidência do evento em AVC isquêmico, lobo parietal e artéria cerebral média.
\end{abstract}

Unitermos. Acidente Vascular Cerebral, Hemiplegia, Postura, Reabilitação.

Citaçáo. Palmini S, Costa J, Grave M. Síndrome de Pusher em Pacientes com AVC e Sua Associação com Gravidade Clínica e Dependência Funcional.

\begin{abstract}
Objective. To establish the prevalence of pusher syndrome (PS) in patients after stroke from clinical criteria contained in Scale for Contraversive Pushing (CPS - evaluation of the symptom of pushing), and correlate them with neurological abnormalities, severity of stroke and functionality. Method. a cross-sectional study with convenience sample of patients of both sexes with a diagnosis of acute stroke. We included clinically stable patients and that were able to assess the severity of the event from the range of National Institute of Health Stroke Scale (NIHSS) and BI. To diagnose the pusher syndrome we used two scoring criteria with different cutoff points in the Scale for Contraversive Pushing: result greater than or equal to 1 (criterion I) or greater than zero (criterion II). Results. 86 patients were evaluated. Mean NIHSS and Barthel index were 8.5 and 48,8 points respectively. The presence of PS was significantly associated with lower values when the Barthel scale, we used the criterion II $(22.5 \pm 8.5$ versus $58.4 \pm 27.3, \mathrm{P}<0.001)$. Conclusion. The prevalence of PS in patients after acute stroke is significant and can vary according to the criteria used. Its presence is associated with clinical severity and functional dependence, higher incidence in ischemic stroke event, parietal lobe and middle cerebral artery, respectively.
\end{abstract}

Keywords. Stroke, Hemiplegia, Posture, Rehabilitation.

Citation. Palmini S, Costa J, Grave M. Patients with Pusher Syndrome and the Association with Clinical Severity and Functional Dependence.

\footnotetext{
A pesquisa foi realizada no hospital Sáo Lucas da Pontifícia Universidade Católica do Rio Grande do Sul (PUCRS), Porto Alegre-RS, Brasil.

1.Fisioterapeuta, Instrutora do Conceito Bobath - IBITA. Mestre em Ciências da Saúde PUCRRS, Porto Alegre-RS, Brasil.

2.Médico, Professor Titular de Neurologia da Faculdade de Medicina e Diretor do Instituto do Cérebro da PUCRS, Porto Alegre-RS, Brasil.

3.Fisioterapeuta, Mestre em Desenvolvimento Regional UNISC; Doutora em Ciências da Saúde PUCRS. Professora dos Cursos de Fisioterapia e Educação Física do Centro Universitário Univates, Lajeado-RS, Brasil.
}

Endereço para correspondência: Magali T. Q. Grave Rua 13 de Maio 230/301, Estrela-RS, Brasil

E-mail: mgrave@univates.br 


\section{INTRODUÇÃO}

Os acidentes cerebrovasculares (AVC), agrupados dentro das causas circulatórias, são, em todo o mundo, a segunda maior causa de óbitos e a primeira causa de incapacidade em adultos jovens. $\mathrm{O}$ fato de estas doenças atingirem pessoas na idade produtiva, além de ter um forte impacto econômico calculado por anos produtivos de vida perdidos, também interfere diretamente na qualidade de vida destas pessoas ${ }^{1}$.

Alterações motoras ocorrem na maioria dos casos, comprometendo, em diferentes graus, a qualidade da postura e dos movimentos normais. Estas alteraçóes, normalmente, estão relacionadas ao comprometimento do tônus muscular, alinhamento biomecânico, e a prejuízos secundários, como deformidades, encurtamentos musculares e neurais, entre outras. Estudos mostram que, além das alterações motoras, outras alteraçōes também comprometem os movimentos e a postura dos pacientes ${ }^{2}$. Pesquisa nesta área tem mostrado que as alteraçôes motoras são apenas uma das partes de um grande sistema de integração, e as sequelas resultantes do AVC dependem da integração entre estes vários sistemas e não ao comprometimento isolado de cada $\mathrm{um}^{2-5}$.

Normalmente, os pacientes hemiplégicos apresentam alteraçôes no controle postural (integração das reaçóes de retificação equilíbrio). Estas alteraçóes ocorrem, pois o controle postural requer a integração intacta dos processos sensoriais e motores e, segundo pesquisas, $88 \%$ de todos os pacientes apresentam algum tipo de hemiparesia e 53\% tem déficit sensorial ${ }^{2,6,7}$. Estas alteraçôes interferem diretamente no desempenho das atividades funcionais dos pacientes.

A síndrome de Pusher ou síndrome do empurrador $^{8}$ é uma alteração do controle postural, descrita clinicamente ${ }^{9-12}$. Este comportamento tem três características típicas: 1) inclinação postural no lado oposto à lesão encefálica com grave desequilíbrio, 2) empurrar fortemente para o lado plégico com o hemicorpo não comprometido, 3) resistência à correção passiva. Autores sugerem que este comportamento está relacionado com uma alteração na percepção postural vertical (PPV), de modo que estes pacientes têm a percepção de que estão alinhados na vertical, quando estáo com aproximadamente $18^{\circ}$ de inclinação para o lado ipsilesional. Ao tentarem ficar na postura em pé ou sentada, o centro de massa desloca-se para o lado ipsilesional e, como resposta, empurram-se para o lado oposto à lesão encefálica ${ }^{10-12}$.

A síndrome de Pusher estará presente em aproximadamente $10 \%$ dos pacientes hemiplégicos e suas consequências interferem, inclusive, no tempo de hospitalização, aumentando em média 29 dias a mais que pacientes com AVC sem síndrome de Pusher ${ }^{6,11,12}$. Pedersen et al. ${ }^{11}$, em estudo consecutivo, prospectivo, ao analisarem 1.197 pacientes com AVC, encontraram uma amostra de 197 sujeitos com SP. Os autores verificaram os resultados da reabilitação, prognóstico funcional e de recuperação destes pacientes, incluindo a função da extremidade superior e aquisição da marcha.

O objetivo deste estudo foi estimar a incidência da síndrome de Pusher em pacientes com AVC agudo e sua associação com a gravidade clínica e dependência funcional.

\section{MÉTODO}

Foi realizado um estudo transversal com pacientes internados no hospital São Lucas da PUCRS, provenientes do Serviço de Neurologia no período de julho a novembro de 2010. A amostra constou de 30 pacientes adultos, selecionados por conveniência, com diagnóstico de AVC agudo, conforme mostra a Tabela 1. Este estudo foi aprovado pelo Comitê de Ética em Pesquisa em seres humanos da PUCRS, sob protocolo de pesquisa registro CEP10/05040, conforme estabelece a Resolução 196/96. Após concordarem em participar, todos os participantes assinaram o Termo de Consentimento Livre e Esclarecido. Para verificação do grau de funcionalidade, foi utilizado o Índice de Barthel ${ }^{13-15}$, que avalia diferentes áreas de função, incluindo alimentação, banho, higiene pessoal, vestir, controle intestinal, controle vesical, transferência para higiene íntima, transferência cadeira e cama, deambulação, subir e descer escadas. A gravidade do AVC foi avaliada através da escala de AVC do NIHSS (National Institute of Health Stroke Scale - NIHSS) ${ }^{16-18}$, instrumento de uso sistemático, que permite uma avaliação quantitativa dos déficits neurológicos relacionados com o AVC. Baseia-se em 11 itens do exame neurológico, comumente afetados pelo AVC, sendo eles: nível de consciência, desvio ocular, paresia facial, linguagem, negligência/extinção, função motora e sensitiva dos membros e 
presença de ataxia. A NIHSS pode ter sua pontuação variando de zero (sem evidência de déficit neurológico) até 42 (paciente em coma e sem resposta). Para diagnóstico da síndrome de Pusher, foi utilizada a SCP - escala de avaliação do sintoma de empurrar - do inglês Scale for Contraversive Pushing ${ }^{19}$. Conforme Karnath et al., a SCP permite avaliar: 1) a simetria da postura espontânea, enquanto sentado e em posição ortostática; 2) a extensão dos membros superiores e/ou inferiores com a superfície de contato, enquanto sentado e em posição ortostática; e 3) a resistência à correção passiva da postura, enquanto sentado e em posição ortostática ${ }^{10,19}$. Têm sido relatadas altas pontuaçôes de confiabilidade intra e interexaminadores $^{20}$. Após a liberação médica, os pacientes foram avaliados, sendo aplicado o protocolo SCP. Foram utilizados dois critérios para o diagnóstico da síndrome de Pusher: o critério I, sugerido por Karnath et al. ${ }^{12}$, tendo como ponto de corte para o diagnóstico o resultado $\geq 1 \mathrm{em}$ cada seção da referida escala. Essa escala consiste em três etapas: A) a primeira é apenas a observação do paciente pelo pesquisador, para verificar a postura corporal de pé e sentado (se simétrica ou assimétrica); B) na segunda, é observado se o paciente permanece com abdução e/ou extensão das extremidades corporais não-paréticas ao movimentar-se, com o objetivo de buscar equilíbrio, sentado e de pé; C) e na terceira é realizada uma resistência passiva pelo pesquisador em seu tórax lateralmente (o paciente é levemente empurrado para o lado), para correçáo da inclinação corporal (caso houver) de pé e sentado. Em cada uma destas etapas, dependendo das reaçóes do paciente, o resultado é anotado e o mesmo recebe uma pontuação, que varia de zero a dois, sendo zero o controle postural conservado e dois a perda do mesmo. O resultado final do teste pode variar entre zero e seis, sendo o resultado mais próximo de zero considerado percepção corporal normal, mais próximo de três, alteração dessa percepção e mais próximo de seis, a perda da mesma. O critério II, sugerido por Baccini et al. ${ }^{19}$ têm como ponto de corte para o diagnóstico, resultado maior que zero em cada sessão da referida escala.

\section{Análise Estatística}

As análises estatísticas foram realizadas no software SPSS versão 17. A descrição das variáveis qualitativas foi realizada por meio das frequências absolutas e relativas; já as variáveis quantitativas foram descritas através da sua média e respectivo desvio padrão.

Para testar as diferenças entre as médias foi utilizado o teste t de Student. Já nos testes de associação entre variáveis qualitativas foi empregado o teste qui-quadrado de Pearson ou o teste exato de Fisher. Em todas as análises considerou-se um nível de significância de 5\%.

\section{RESULTADOS}

Foram selecionados 86 pacientes, e destes, 30 foram incluídos. Dos 56 excluídos, 50 não apresentavam condições cognitivas para responderem aos comandos verbais da pesquisadora, ficando impossibilitados de participarem das avaliações. Os demais (06) não assinaram TCLE.

Com relação às características demográficas e clínicas dos participantes, 17 são do sexo masculino, com

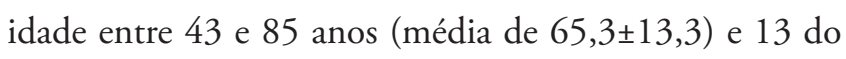
sexo feminino, com idade entre 30 e 85 anos (média de $66,6 \pm 17,4)$. Considerando o tipo de AVC, $87 \%$ dos pacientes avaliados apresentaram AVC isquêmico. Os pacientes com AVC hemorrágico tiveram escore médio de 9,5 na escala NIHSS e escore médio de 21,2 no IB (Tabela 1). $86 \%$ dos pacientes foram acometidos por AVC isquêmico, 53,3 pacientes apresentaram hemiplegia em hemicorpo direito e a artéria cerebral média (ACM) foi acometida em $70 \%$ dos casos. O lobo parietal foi comprometido em $60 \%$ dos pacientes. A média encontrada na escala NIHSS foi de 8,5 pontos e a média do IB foi de 48,8 (Tabela 2). Quando utilizado o critério II, observa-se uma associação entre o diagnóstico de SP e o tipo de AVC, havendo uma maior associação com AVC isquêmico $(62,5 \%, p=0,048)$. Não foi observada associaçáo significativa entre o diagnóstico da SP com o sexo e lateralização da lesão encefálica (Tabela 3). Quando utilizado o critério I, não foi observado diferença entre as variáveis estudadas nos pacientes em relação a apresentar ou não apresentar a síndrome. O critério II mostrou-se mais sensível, apresentando oito pacientes com a síndrome. Conforme a literatura descreve, houve maior chance de ter a síndrome os pacientes acometidos por AVC isquêmico (Tabela 4). 
Tabela 1

Características demográficas e clinicas dos pacientes incluidos $(n=30)$

\begin{tabular}{|c|c|c|c|c|c|c|}
\hline PCTE & IDADE & GÊNERO & $\begin{array}{c}\text { HEMIPLEGIA } \\
\text { D/E }\end{array}$ & TIPO AVC & NIHSS & BARTHEL \\
\hline 1 & 61,0 & M & $\mathrm{D}$ & I & 11,0 & 55,0 \\
\hline 2 & 58,0 & $\mathbf{F}$ & $\mathbf{E}$ & $\mathbf{H}$ & 9,0 & 25,0 \\
\hline 3 & 61,0 & $\mathbf{M}$ & $\mathbf{E}$ & $\mathbf{H}$ & 13,0 & 15,0 \\
\hline 4 & 85,0 & $\mathrm{~F}$ & E & I & 12,0 & 25,0 \\
\hline 5 & 86,0 & M & $\mathrm{D}$ & I & 4,0 & 90,0 \\
\hline 6 & 72,0 & M & $\mathrm{D}$ & I & 0,0 & 100,0 \\
\hline 7 & 79,0 & M & E & I & 3,0 & 90,0 \\
\hline 8 & 30,0 & $\mathrm{~F}$ & $\mathrm{D}$ & I & 0,0 & 70,0 \\
\hline 9 & 72,0 & $\mathrm{~F}$ & E & I & 6,0 & 65,0 \\
\hline 10 & 74,0 & F & $\mathrm{E}$ & I & 0,0 & 95,0 \\
\hline 11 & 33,0 & M & E & I & 12,0 & 40,0 \\
\hline 12 & 71,0 & $\mathbf{M}$ & D & I & 12,0 & 35,0 \\
\hline 13 & 49,0 & M & $\mathrm{D}$ & I & 12,0 & 20,0 \\
\hline 14 & 66,0 & F & D & I & 21,0 & 25,0 \\
\hline 15 & 63,0 & $\mathbf{M}$ & D & I & 11,0 & 25,0 \\
\hline 16 & 65,0 & F & $\mathrm{E}$ & $\mathrm{H}$ & 10,0 & 35,0 \\
\hline 17 & 63,0 & M & $\mathrm{D}$ & I & 11,0 & 60,0 \\
\hline 18 & 85,0 & M & E & I & 1,0 & 45,0 \\
\hline 19 & 43,0 & $\mathrm{~F}$ & $\mathrm{E}$ & I & 4,0 & 80,0 \\
\hline 20 & 76,0 & M & $\mathrm{D}$ & I & 5,0 & 35,0 \\
\hline 21 & 77,0 & F & $\mathrm{D}$ & I & 2,0 & 80,0 \\
\hline 22 & 80,0 & F & $\mathrm{D}$ & I & 10,0 & 30,0 \\
\hline 23 & 59,0 & $\mathbf{M}$ & $\mathbf{E}$ & $\mathbf{H}$ & 6,0 & 10,0 \\
\hline 24 & 63,0 & $\mathrm{M}$ & $\mathrm{D}$ & I & 24,0 & 25,0 \\
\hline 25 & 80,0 & F & $\mathrm{D}$ & I & 15,0 & 30,0 \\
\hline 26 & 46,0 & $\mathrm{~F}$ & $\mathrm{D}$ & I & 15,0 & 35,0 \\
\hline 27 & 51,0 & $\mathbf{M}$ & $\mathbf{E}$ & I & 8,0 & 30,0 \\
\hline 28 & 71,0 & M & E & I & 0,0 & 90,0 \\
\hline 29 & 67,0 & M & $\mathrm{D}$ & I & 0,0 & 90,0 \\
\hline 30 & 85,0 & $\mathbf{F}$ & $\mathbf{E}$ & I & 18,0 & 15,0 \\
\hline
\end{tabular}

$\mathrm{M}=$ masculino, $\mathrm{F}=$ feminino, $\mathrm{D}=$ direita, $\mathrm{E}=$ esquerda, $\mathrm{I}=\mathrm{isquemico}, \mathrm{H}=$ hemorrágico, NIHSS=National Institute of Health Stroke Scale. Em negrito pacientes com diagnóstico de Síndrome de Pusher

\section{DISCUSSÃO}

O AVC é um importante problema de saúde pública no mundo. Suas consequências podem ser de grande impacto, gerando enormes demandas de recursos em diagnóstico, tratamento e reabilitação ${ }^{21-24}$. No Brasil, o AVC é responsável por altas taxas de mortalidade, invalidez e elevado custo social e econômico ${ }^{5,24}$. Cresce a preocupação em relação à limitação funcional destes pacientes, que geralmente ficam incapazes de exercer suas atividades de vida normal, gerando a necessidade de uma abordagem mais específica pelos profissionais da área $\mathrm{da}$ saúde. Neste sentido, faz-se necessário aprofundar o conhecimento, utilizando novos recursos e abordagens para o diagnóstico e tratamento destas incapacidades ${ }^{5,23,24}$.

Um dos problemas ainda pouco considerado, que interfere negativamente na recuperaçáo funcional destes pacientes, é a síndrome de Pusher, onde os mecanismos causadores têm sido relacionados a alteraçóes de percep- 
Tabela 2

Características dos pacientes incluidos em relação às variáveis estudadas

\begin{tabular}{lc}
\hline Variável & Média ou $\%$ \\
\hline Idade & 65,7 \\
Homens & $56,6 \%$ \\
Hemiplegia Dir. & $53,3 \%$ \\
AVC I & $86,6 \%$ \\
AVC H & $13,3 \%$ \\
Território Cerebral & \\
ACA & $6,6 \%$ \\
ACM & $70 \%$ \\
ACP & $23,3 \%$ \\
Lobo Cerebral & \\
Parietal & $63,3 \%$ \\
Occipital & $6,6 \%$ \\
Frontal & $26,6 \%$ \\
Temporal & $3,3 \%$ \\
Barthel & 48,8 \\
NIHSS & 8,5 \\
\hline
\end{tabular}

AVC I=acidente vascular isquêmico, $\mathrm{AVCH}=$ acidente vascular hemorrágico; $\mathrm{ACA}=$ artéria cerebral anterior; $\mathrm{ACM}=$ artéria cerebral média; $\mathrm{ACP}=$ artéria cerebral posterior; DIR=direita.

Os dados são apresentados como média ou contagem (\%). NIHSS: média 14,5; SD 6,65, Barthel: mediana 35; intervalo interquartil 25.

Tabela 3

Diagnóstico da sindrome de Pusher, segundo critérios estabelecidos com diferentes pontos de corte: critério 1 ou 2

\begin{tabular}{ccc}
\hline $\begin{array}{c}\text { Critério } \\
\text { SCP }\end{array}$ & Diagnóstico & $\begin{array}{c}\text { Pacientes com diagnóstico } \\
\text { clínico SP }\end{array}$ \\
\hline Critério 1 & Negativo & 29 \\
Positivo & 01 & $3,3 \%$ \\
Critério 2 & 22 & 22 \\
Positivo & 08 & $26,6 \%$ \\
\hline
\end{tabular}

$\mathrm{SCP}=$ Scale Controversive Pusher; $\mathrm{SP}=$ Síndrome de Pusher

Critério 1: resultado $\geq 1 \mathrm{em}$ cada seçấo da escala,Critério 2: resultado $>0 \mathrm{em}$ cada seção da escala

ções de verticalidade, sendo o comportamento observado, consequência de um conflito sensorial. Perrennou et al. (2008) analisou os mecanismos subjacentes atribuídos à discifunção da prcepção vertical que leva aço comportamento reativos destes pacientes. No entanto, as alterações na percepção da verticalidade destes pacientes ainda não são claras ${ }^{10,11,25}$. Embora ainda existam poucos dados disponíveis em relação a esta síndrome, alguns estudos mostram que ela pode impedir, ou pelo menos retardar, a recuperação funcional, equilíbrio e e marcha dos pacientes ${ }^{8,10,19}$. No presente estudo, o termo controle postural foi utilizado como o somatório das reações de equilíbrio, retificação e proteção, normalmente alteradas nas lesóes do $\mathrm{SNC}^{10,26}$.

Sobre o impacto da síndrome de Pusher na reabilitação dos pacientes, um estudo determinou que a síndrome de Pusher não era verdadeiramente uma síndrome, visto que o lado da lesão, a negligência no lado oposto à lesão encefálica e anosognosia não estavam relacionados ao comportamento de empurrar ${ }^{11}$. Diferentes nomes utilizados para descrever esta síndrome incluem lateropulsão, contraversive pushing (empurrar para o lado contrário à lesão) e ipsilateral pushing (empurrar para o hemicorpo afetado). Lateropulsão é o termo mais específico para descrever a inclinação tônica postural no plano frontal do suporte vertical ${ }^{11}$.

Apesar do aumento do número de estudos relacionados a esta síndrome, ainda existe muita discordância entre os pesquisadores quanto à prevalência, fisiopatologia e tratamento. Provavelmente, um dos motivos importantes para justificar esta divergência seja a falta de consenso em relação ao critério utilizado para o diagnóstico clínico $^{12,19}$. Neste estudo, utilizou-se para diagnóstico da síndrome de Pusher um instrumento de avaliação desenvolvido por Karnath et al. ${ }^{19}$.

Há relatos na literatura da presença da síndrome de Pusher em aproximadamente $10 \%$ dos pacientes hemiplégicos, sem significativas associações entre o local, tipo da lesão e a presença da mesma ${ }^{11,12}$.

O presente estudo mostrou que a síndrome de $\mathrm{Pu}$ sher ocorreu em 26,6\% dos pacientes incluídos na amostra quando utilizado o critério II para diagnóstico. Quando utilizado o critério I, apenas um paciente apresentou o diagnóstico. $\mathrm{Na}$ literatura, ainda existe divergência em relação à prevalência da síndrome de Pusher nos pacientes acometidos por AVC, provavelmente devido aos diferentes pontos de corte estabelecidos para o diagnóstico ${ }^{19}$. A utilização do critério I, por ser um critério muito conservador, poderá levar a resultados falso negativos, enquanto que a utilização do critério II, por ser um critério mais abrangente, poderá levar a resultados falso positivos ${ }^{19}$.

Tem sido descrita a associação entre a síndrome de Pusher e a lesão no hemisfério cerebral direito com comprometimento em lobo parietal ${ }^{10-12}$. Atualmente, sabe-se que a síndrome de Pusher pode estar presente em pacien- 
Tabela 4

Comparaçâo de características de pacientes com e sem sindrome de Pusher de acordo com dois critérios de positividade da SCP

\begin{tabular}{|c|c|c|c|c|c|c|}
\hline \multirow[b]{2}{*}{ Variável } & \multicolumn{3}{|c|}{ Critério 1} & \multicolumn{3}{|c|}{ Critério 2} \\
\hline & $\begin{array}{l}{[+]} \\
n=1\end{array}$ & $\begin{array}{c}{[-]} \\
\mathbf{n}=29\end{array}$ & $\mathbf{P}$ & $\begin{array}{c}{[+]} \\
n=8\end{array}$ & $\begin{array}{c}{[-]} \\
\mathbf{n}=22\end{array}$ & $\mathbf{P}$ \\
\hline Idade, anos & $71,0 \pm$ nd & $65,6 \pm 15,2$ & 0,725 & $64,2 \pm 10,2$ & $66,2 \pm 16,5$ & $0,316[1]$ \\
\hline Sexo masculino, $\mathrm{n}^{\circ}(\%)$ & $1(100,0)$ & $16(55,2)$ & 0,999 & $5(62,5)$ & $12(54,5)$ & $0,999[2]$ \\
\hline \multicolumn{7}{|l|}{$\begin{array}{c}\text { Lateralizaçáo da } \\
\text { hemiplegia, no (\%) }\end{array}$} \\
\hline Direita & $1(100,0)$ & $15(51,7)$ & 0,999 & $3(37,5)$ & $13(59,1)$ & $0,417[2]$ \\
\hline Esquerda & $0(0,0)$ & $14(48,3)$ & & $5(62,5)$ & $9(40,9)$ & \\
\hline \multicolumn{7}{|l|}{ Tipo de AVC, no (\%) } \\
\hline Isquêmico & $1(100,0)$ & $25(86,2)$ & 0,999 & $5(62,5)$ & $21(95,5)$ & $0,048[2]$ \\
\hline Hemorrágico & $0(0,0)$ & $4(13,8)$ & & $3(37,5)$ & $1(4,5)$ & \\
\hline NIHSS & $12,0 \pm \mathrm{nd}$ & $8,4 \pm 6,6$ & 0,592 & $12,3 \pm 5,1$ & $7,1 \pm 6,5$ & $0,054[1]$ \\
\hline Barthel & $35,0 \pm$ nd & $49,3 \pm 29,0$ & 0,485 & $22,5 \pm 8,5$ & $58,4 \pm 27,3$ & $<0,001[1]$ \\
\hline
\end{tabular}

$\mathrm{SCP}=$ Scale Contraversive Pusher; AVC=Acidente Vascular Cerebral, NIHSS=Natioanl Institute of Health Stroke Scale;

[1] teste T de student [2] teste qui-quadrado de Pearson ou exato de Fisher.

tes com outros acometimentos cerebrais, como traumatismo craniano. Observa-se que a síndrome de Pusher pode não estar apenas em uma área específica e sim envolvida a uma rede neural, muitas vezes envolvendo o tálamo e suas conexôes ${ }^{27}$. Estudos mais recentes revelaram que o tálamo posterior é uma importante parte do sistema relacionado à percepção da orientação do corpo $^{24,28}$.

Em estudo que avaliou 21 pacientes com diagnóstico de SP pós AVC e lesões corticais sem comprometimento talâmico, foi possível constatar que as áreas corticais mais comprometidas foram o giro pós-central e a ínsula. Considerando o comprometimento do giro pós - central (lobo parietal), estes achados coincidem com a nossa observação de comprometimento do lobo parietal e do território da artéria cerebral média ${ }^{29}$.

Assim, os seguintes ramos da artéria cerebral média poderiam estar envolvidos na síndrome de Pusher pelo território comprometido nesta patologia ${ }^{28}$ :

1. Artéria candelabra, opérculo frontal ou pré-rolândica, que irriga primariamente a ínsula, opérculo, a área de Broca e córtex adjacente

2. Artéria do sulco central que supre o giro motor e sensorial.

3. Artéria parietal posterior, artéria angular e artéria temporal posterior. A artéria parietal posterior irriga a porção inferior do lobo parietal e giro su- pra marginal. A artéria angular que supre o giro angular e a área parieto-occipital, e artéria temporal posterior que vasculariza a porção posterior do lobo temporal.

Neste estudo, não houve relação com o hemisfério cerebral lesado, porém, 63,3\% dos pacientes apresentaram lesão no lobo parietal, comparável aos dados da literatura ${ }^{10,12}$. Quanto ao acometimento do território cerebral, observou-se comprometimento das artérias cerebral média, cerebral anterior e cerebral posterior em $70 \%$, $6,6 \%$, e $23,3 \%$, respectivamente.

Pesquisa sobre a recuperação do controle postural pós - AVC com 78 pacientes consecutivos observou que, em $91 \%$ dos casos, o tipo de AVC foi isquêmico, próximo dos $86,2 \%$ da nossa casuística. O fato de a maioria dos pacientes apresentarem comprometimento no lobo parietal e hemiplegia pode ser justificado pelo comprometimento mais frequente no território da artéria cerebral média, o que acometeu em $70 \%$ dos pacientes deste estudo $^{30}$.

Encontramos, em nossos resultados, uma associação entre a síndrome de Pusher com a gravidade do AVC e dependência funcional avaliada através da escala de Barthel e de NIHSS. Quando considerado o critério II, esta associação foi significativa para dependência funcional e tendeu à significância estatística para a gravidade clínica 
do AVC, conforme resultados apresentados nas tabelas 3 e 4. A falta de associação significativa quando utilizado o critério I deveu-se à falta de poder da amostra, pois apenas um paciente foi diagnosticado como portador da síndrome de Pusher. Tal achado poderia ter implicações práticas, pois indicaria que pacientes portadores da síndrome deveriam ter uma abordagem terapêutica diferenciada diante de um provável prognóstico mais reservado de recuperação funcional.

Estudos sobre o comprometimento das habilidades funcionais de pacientes com síndrome de Pusher pós-AVC utilizaram o Chedoke-Mcmaster Disability Inventory (CRDI), o Physiotherapy Clinical Outcome Variables (COVS) e o Functional Independence Measure (FIM) para avaliação, e evidenciaram que estes pacientes apresentaram menor recuperaçáo motora e da habilidade funcional aos três meses pós-AVC comparado aos pacientes sem síndrome de Pusher ${ }^{27-31}$.

Em um estudo retrospectivo incluindo 36 pacientes com AVC e com lateropulsão, termo que utilizaram como sinônimo de síndrome de Pusher, os autores verificaram que a independência funcional foi mais baixa, quando utilizado o Functional Independence Measure $(\mathrm{FIM})^{27}$.

Estes achados são semelhantes com a nossa observação quanto ao maior comprometimento funcional encontrado nos pacientes portadores de síndrome de Pusher. Entretanto, estudo realizado na Dinamarca (The Copenhagen Stroke Study, 1996) observou que não houve diferença na recuperação dos pacientes com síndrome de Pusher pós - AVC quando comparados aos pacientes sem a síndrome, embora o processo de recuperação tenha sido consideravelmente mais lento ${ }^{11}$.

Estudos demonstram que o diagnóstico da síndrome de Pusher pode estar presente em pacientes com hemiplegia/hemiparesia leve, embora seja gravemente incapacitante ${ }^{10,28}$. A partir desta associação, pode-se evidenciar a importância do diagnóstico e conhecimento desta síndrome por profissionais da área da saúde, pois, apesar da SP poder ser transitória, seus sinais e sintomas clínicos deveriam ser minimizados o mais precocemente possível, através de abordagem específica de tratamento, para diminuir os prejuízos relacionados à funcionalidade destes pacientes ${ }^{10}$. Estudos mostram que, embora o paciente apresente a síndrome de Pusher associada ao comprometimento funcional grave, poderá haver uma recuperação funcional após a resolução da síndrome ${ }^{11,13}$.

\section{CONCLUSÃO}

Nossos resultados demonstram que a síndrome de Pusher é um transtorno que ocorre após um episódio de AVC, sendo que sua prevalência depende dos critérios clínicos utilizados. A artéria cerebral média e o lobo parietal foram mais acometidos nos nossos pacientes. A etiologia do AVC mais frequentemente associada à síndrome de Pusher foi a isquêmica. No entanto, sua associação com a gravidade do evento e o grau de dependência funcional indica que existe relevância clínica em seu diagnóstico. Torna-se necessário o desenvolvimento de critérios padronizados para sua definição e estudos longitudinais para que se possam delinear estratégias de intervenção.

\section{REFERÊNCIAS}

1.Murray CJ, Lopez AD. Mortality by cause for eight regions of the world: Global burden of disease study. Lancet 1997;349(9061):1269-76.

http://dx.doi.org/10.1016/S0140-6736(96)07493-4

2.Donnan GA, Fisher M, Macleod M, Davis SM. "Stroke." Lancet 2008;371(9624):1612-23.

http://dx.doi.org/10.1016/S0140-6736(08)60694-7

3.World Health Organization. Deaths by cause, sex and mortality stratum in WHO regions, estimates for 2002. Geneva: World Health Organization, 2004, p.141-149.

4.Falcão IV, Carvalho EMF, Barreto KML, Lessa FJD, Leite VMM. Acidente Vascular Cerebral Precoce: Implicaçôes para adultos em idade produtiva atendidos pelo sistema único de saúde. Rev. Bras Saúde Matern Infant 2004;4(1):95-102.

http://dx.doi.org/10.1590/S1519-38292004000100009

5.Dobkin B. The economic impact of stroke. Neurology 1995;45(Suppl 1):S6S9.

6.Ellekjaer H, Holmen J, Indredavik B, Terent A. Epidemiology of stroke in Innherred, Norway, 1994 to 1996 . Incidence and 30 day case fatality rate. Stroke 1997;28:2180-4.

http://dx.doi.org/10.1161/01.STR.28.11.2180

7.Senelick RC, Rossi PW, Dougherty K. Living with stroke: A guide for families. Chicago: Contemporary books, 1994, p.38-45.

8.Santos-Pontelli TEG, Pontes Neto O, Araújo DB. Controle postural na síndrome de Pusher: influência dos canais semicirculares laterais. Rev Bras Otorrinolaringol 2005;71(4):448-52.

http://dx.doi.org/10.1590/S0034-72992005000400008

9.Beevor C. Remarks on paralysis of movement of the trunk in hemiplegia, and the muscles which are affected. BMJ 1909;1(2519):881-5.

http://dx.doi.org/10.1136/bmj.1.2519.881 
10.Davies PM. Steps to follow a guide to the treatment of adult hemiplegia. 2nd. ed. Berlin: Springer-Verlag, 2000, p.101-103.

http://dx.doi.org/10.1007/978-3-642-57022-3

11.Pedersen PM, Wandel A, Jørgensen HS, Nakayama H, Raaschou HO, Olsen TS. Ipsilateral Pushing in Stroke: Incidence, Relation to Neuropsychological Symptoms, and Impact on Rehabilitation. The Copenhagen Stroke Study. Arch Phys Med Rehabil 1996;77:25-8. http://dx.doi.org/10.1016/S0003-9993(96)90215-4

12.Karnath HO, Ferber S, Dichgans J. The neural representation of postural control in humans. Proc Natl Acad Sci 2000;(25):13931-6.

http://dx.doi.org/10.1073/pnas.240279997

13. Mahoney FI, Barthel DW. Functional evaluation: the Barthel Index. Md State Med J 1965;14:61-5.

14.Collin C, Davies S, Horne V. Functional Evaluation: the Barthel Index."; Md State Med J 1988;14:61-5.

15. Walter J, Grave MTQ, Perico E. Avaliação das habilidades psicomotoras e motricidade global em paciente portadora da doença de Huntington. ConScientia e Saúde 2009;8(4):655-63.

16.Adams HP Jr, Davis PH, Leira EC, Chang KC, Bendixen BH, Clarke WR, et al. Baseline NIH Stroke Scale score strongly predicts outcome after stroke: A report of the Trial of Org 10172 in Acute Stroke Treatment (TOAST). Neurology 1999;53:126-31.

http://dx.doi.org/10.1212/WNL.53.1.126

17.Lyden P, Brott T, Tilley B, Welch KM, Mascha EJ, Levine S, et al. "Improved reliability of the NIH Stroke Scale using video training." Stroke 1994;25:2220-6.

http://dx.doi.org/10.1161/01.STR.25.11.2220

18.Martins SCO, Brondani R, Friederich M. Quatro anos de experiência no tratamento trombolítico do AVC isquêmico na cidade de Porto Alegre.Neurociências 2006;14(1):31-6.

19.Baccini M, Paci M, Nannetti L, Biricolti C, Rinaldi LA. Scale for Contraversive Pushing:Cutoff Scores for Diagnosing "Pusher Behavior" and Construct Validity. Physical Therapy 2008;88(8):947-55.

http://dx.doi.org/10.2522/ptj.20070179

20.Lagerqvist J, Skargren E. Pusher syndrome: reliability, validity andsensitivity to change of classification instrument. Advances in Physiotherapy 2006;8(4):154-60.

http://dx.doi.org/10.1080/14038190600806596
21.Karnath HO, Ferber S, Dichgans J. The origin of contraversive pushing:evidence for a second graviceptive system in humans. Neurology 2000;55:1298-1304.

http://dx.doi.org/10.1212/WNL.55.9.1298

22.Pontes-Neto O, Silva GS, Feitosa MR. Stroke awareness in Brasil: results in a community-based study. Stroke 2008;39:292-6.

http://dx.doi.org/10.1161/STROKEAHA.107.493908

23.Gillum RF, Sempos CT. The end of the long-term decline in stroke mortality in the United States? Stroke 1997;28:1527-9.

http://dx.doi.org/10.1161/01.STR.28.8.1527

24.Pires SL, Gagliardi JR, Gorzoni LM. Estudo das freqüências dos principais fatores de risco para acidente vascular cerebral isquêmico em idosos. Arq Neuropsiquiatr 2004;62(3-B):844-51.

http://dx.doi.org/10.1590/S0004-282X2004000500020

25.Perennou DA, Mazibrada G, Chauvineau V, Greenwood R, Rothwell J, Gresty MA, et al. Lateropulsion, pushing and verticality perception in hemisfhere stroke: a causal relationship? Brain 2008 Sep;131(9):2401-13.

http://dx.doi.org/10.1093/brain/awn170

26. Rayerson SD. Reabilitação Neurológica. 4.ed. São Paulo: Manole, 2004, p.73-76.

27.Ticini, LF, Klose U, Karnath HO. Perfusion imaging in pusher syndrome to investigate the neural substrates involved in controlling upright body position. PloS One 2009; 4(5):5737.

http://dx.doi.org/10.1371/journal.pone.0005737

28.Santos-Pontelli TE, Pontes-Neto OM, Colafêmina JF, Araújo DB, Santos AC, Leite JP. Pushing Behavior and Hemiparesis - Which is Critical for Functional Recovery in Pusher Patients? Arq Neuropsiquiatr 2007;65(2-B):536-9. http://dx.doi.org/10.1590/S0004-282X2007000300035

29.Johannsen L, Broetz D, Karnath HO. Pusher syndrome"following cortical lesions that spare the thalamus. J Neurol 2006;253:455-63.

http://dx.doi.org/10.1007/s00415-005-0025-7

30. Waddington MM, Ring AB. Syndromes of occlusions of middle cerebral artery branches - angiographic and clinical correlation. Brain 1968;91(4):685-96. http://dx.doi.org/10.1093/brain/91.4.685

31.Van Nes WJI. Balance recovery after supratentorial stroke - influence of hemineglect and the effects of somatosensory stimulation. Thesis, Radboud University Nijmegen, 2009, p.28-31. 